\title{
Exploring Structural Implications of diphosphinamine ligands in Medicine and Catalysis
}

\author{
Dumisani Vincent Kama ${ }^{1 *}$, Alice Brink ${ }^{1}$, Roger Alberto ${ }^{2}$, Andreas Roodt $^{1}$ \\ ${ }^{1}$ Department of Chemistry, University of the Free State, PO Box 339, Bloemfontein, 9300; \\ ${ }^{2}$ Institute for Inorganic Chemistry, University of Zürich, Winterthurstrasse, Zürich, Switzerland.
}

tomkama112@gmail.com

Phosphine ligands are considered by many as one of the most significant class of ligands in organometallic chemistry. The search of new phosphine chelators, as well as the subsequent functionalization thereof, is a continuing process in order to induce appropriate properties for highly effective catalyst and to a lesser extend in medicinal purposes. Of particular interest is the search for watersoluble and highly stable ligands that can preserve their aquatic solubility even after metal coordination.

In this study, we aim to improve the efficiency of middle/late transition metal homogeneous catalysts (i.e. $\mathrm{Re}, \mathrm{Rh}, \mathrm{Pd}$ and $\mathrm{Pt}$ ) and fac$\left[\mathrm{M}(\mathrm{CO})_{3}\right](\mathrm{M}=\mathrm{Re}$ and $\mathrm{Tc})$ radiopharmaceutical synthons by selectively introducing monodentate and bidentate phosphine ligands consisting of various electronic and steric properties. The use of systematically altered bidentate phosphine ligands such as diphosphinoamine ligands has already been reported to show high selectivity improvements in catalytic reactions such as ethylene triand tetramerization [1].

A series of diphosphinoamine ligands was synthesized using methods described in literature [2, 3]. These ligands were then coordinated to various metal (i.e. $\operatorname{Re}(\mathrm{I}), \mathrm{Tc}(\mathrm{I}), \mathrm{Pt}(\mathrm{II})$ and $\mathrm{Pd}(\mathrm{II})$ ). Results obtained from the biological analysis and catalytic evaluations have opened up a new window of opportunities for such compounds.

[1] Cloete, N., Visser, H.G., Engelbrecht, I., Overett, M.J., Gabrielli, W.F \& Roodt, A. (2013). Inorg. Chem, 52(5) $2268-2270$.

[2] Engelbrecht, I., Visser, H.G \& Roodt, A. (2012). Acta Cryst, E. 68, m916-m917.

[3] Cloete, N., Visser, H.G., Roodt, A., Dixon, J.T \& Blann, K. (2008), Acta Cryst. E. 64, o480.

Keywords: Diphosphinoamine, Catalysis, Radiopharmaceutical

Financial assistance from the University of Zurich and the University of the Free State is gratefully acknowledged. We thank the Swiss National Science Foundation and the South African National Research Foundation for financial support. We also express our gratitude towards SASOL, PETLabs Pharmaceuticals, for financial support of this project. 